\title{
Respuesta Agronómica de Dos Variedades de Papa (spp. tuberosum y andigena) a Diferentes Niveles de Fertilización Mineral
}

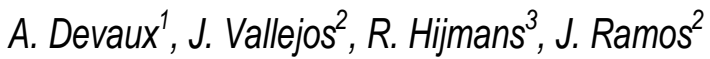

\begin{abstract}
Resumen
Durante las campañas de 1990-91 y 1991-92, se estudió en la Estación Experimental Toralapa en Cochabamba, Bolivia, la respuesta agronómica de dos variedades de papa, Rosita (spp. tuberosum) y Waych'a (ssp. andigena) a cuatro niveles de nitrógeno y fósforo combinados con dos niveles de potasio. Se observó una respuesta marcada de ambas variedades a la aplicación de nitrógeno y fósforo pero el efecto del potasio sobre el rendimiento no fue significativo Las dos variedades presentaron resultados diferentes en los dos años de prueba mostrando la importancia del efecto varietal y de las condiciones agoclimáticas sobre la respuesta a niveles de fertilización. Los datos de análisis de suelos indican que Waych'a extrae menos fósforo del suelo que Rosita. La relación entre el rendimiento y la duración de cobertura del suelo por el follaje mostró la respuesta de Rosita a niveles de fertilización más altos que Waych'a y parece indicar una mayor eficiencia de Rosita para utilizar la radiación interceptada en la producción de tubérculos.
\end{abstract}

Palabras claves adicionales: nitrógeno, fósforo, cobertura de suelo, intercepción de luz, Bolivia.

Aceptado para publicación: agosto 1997.

1 Investigador del CIP, IBTA-PROJNPA, Casilla 4285, Cochabamba, Bolivia.

2 Investigador, Ingeniero Agrónomo, IBTA-PROINPA, Casilla 4285, Cochabamba, Bolivia.

3 Investigador del CIP, Casilla 1558, Lima 100, Perú 


\title{
Agronomic Response of Two Potato Varieties (spp. tuberosum and andigena) at Different Levels of Mineral Fertilization
}

\begin{abstract}
Summary
During the 1990-91 and 1991-92 growing seasons trials were carried out at the Toralapa Experimental Research Station in Cochabamba, Bolivia to study the response of two varieties of potato, Rosita (spp. tuberosum) and Waych'a (spp. andigena) to four levels of nitrogen and phosphorus combined with two levels of potassium. Both varieties responded well to the application of nitrogen and phosphorus, but there was no significant yield difference due to the application of potassium. Both varieties gave different results during the two years of evaluation showing the importance of agroclimatic conditions on the effect of different levels of fertilization. It is clear from the soil data analysis that Waych'a extracts less phosphorus from the soil than Rosita. The relation between foliage soil coverage duration and yield data showed a response of Rosita to higher levels of fertilization than Waych'a, and seem to indicate a greater efficiency in Rosita to transform intercepted radiation into tuber production.
\end{abstract}

Additional index words: nitrogen, phosphorus, ground cover, light interception, Bolivia.

\section{Introducción}

En la mayoría de las zonas de producción, la baja fertilidad de los suelos es uno de los factores que limita la producción de papa en Bolivia $(5,6,8)$. Considerando que las estrategias de fertilización del cultivo de la papa deben concebirse en base a las condiciones de fertilidad de los suelos de las zonas de producción y de los sistemas de producción manejados por los agricultores, el Programa de Investigación de la Papa (PROINPA) inició una serie de trabajos de investigación para desarrollar propuestas de fertilización del cultivo de la papa que respondan a las condiciones agroeconómicas de los sistemas de producción de Bolivia. Estos trabajos incluyeron estudios 
agroeconómicos sobre la respuesta de variedades de diferentes subespecies a niveles de fertilización, la combinación de prácticas de fertilización orgánica e inorgánica y sus efectos remanentes en los cultivos de la rotación y estudios sobre el uso de abonos verdes. Se analizan también la influencia de estas prácticas sobre la infección del cultivo de papa por nematodos.

Este artículo presenta los resultados del análisis de la respuesta agronómica de dos variedades, una de la subespecie tuberosum y otra de andigena a la fertilización con niveles distintos de los tres elementos principales nitrógeno, fósforo y potasio. Además de observar las diferencias en rendimiento, este estudio contribuye a explicar estas diferencias entre variedades. Esta investigación se realizó en colaboración con el Proyecto FERTISUELOS (FAO-SNAG). También se realizó un análisis económico de la respuesta de estas dos variedades a niveles de fertilización mineral, analizando diferentes estrategias de inversión en el cultivo de acuerdo a los tipos de agricultores y los riesgos ligados al cultivo. Este análisis se presentará en otro artículo.

\section{Materiales y Métodos}

Dos ensayos fueron conducidos durante las campañas agrícolas 1990-91 y 1991-92 en la Estación Experimental Toralapa del Instituto Boliviano de Tecnología Agropecuaria (IBTA), del departamento de Cochabamba, situada a una altura de $3,430 \mathrm{msnm}$, en los $17^{\circ} 25^{\prime}$ de latitud sur y $65^{\circ} 61^{\prime}$ de longitud oeste. Los promedios de las temperaturas máxima, mínima y media durante este período fueron de $15^{\circ} \mathrm{C}, 1^{\circ} \mathrm{C}$ y $9^{\circ} \mathrm{C}$, respectivamente. Las precipitaciones en promedio de los últimos 11 años y las observadas durante las dos campañas de realización de los ensayos, se presentan en la Figura 1.

Los ensayos se realizaron con las variedades Waych'a (Solanum tuberosum spp. andigena) y Rosita (Solanum tuberosum spp. tuberosum). En ambas campañas se utilizaron tubérculos-semillas $(35-45 \mathrm{~mm})$ que fueron sembrados a una densidad de $0.7 \mathrm{~m}$ entre surcos y $0.3 \mathrm{~m}$ entre plantas. 


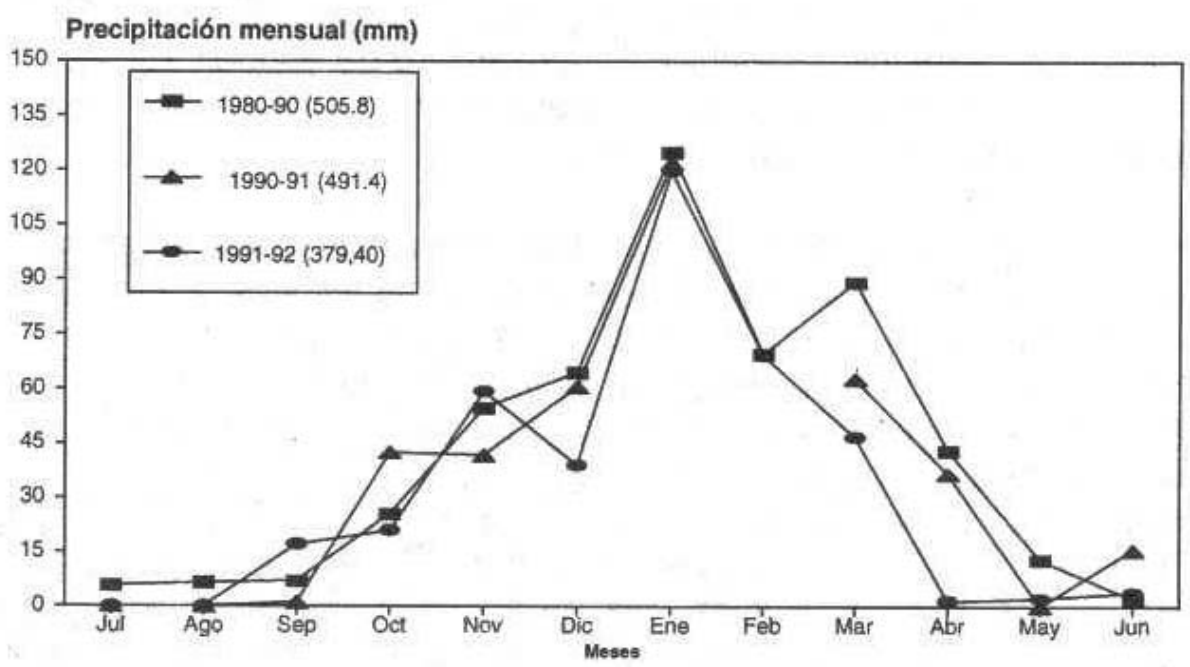

Figura 1. Precipitación mensual de dos campañas agrícolas (1990-91 y 1991-92) y promedio de las precipitaciones mensuales en 11 años (1980-1990) en la Estación Experimental Toralapa.

En las dos campañas, cuatro niveles de nitrógeno $(\mathrm{N})$ y de fósforo $\left(\mathrm{P}_{2} \mathrm{O}_{5}\right), 0$, 80, 160 y $240 \mathrm{Kg}$. por hectárea, fueron combinados para cada variedad en un arreglo factorial $4 \times 4$, bajo el diseño de Bloques Randomizados. Además se evaluó el efecto de la aplicación de tres niveles de potasio $\left(\mathrm{K}_{2} \mathrm{O}\right), 0,60,120$ $\mathrm{Kg}$. por hectárea, que fueron combinados sólo con los niveles de 160 y 240 $\mathrm{Kg} . / \mathrm{ha}$ de nitrógeno y de $160 \mathrm{Kg}$./ha de fósforo (Tabla 1). Para analizar el efecto de la aplicación del potasio se consideró un diseño de Bloques Randomizados con arreglo factorial $2 \times 3$.

Las fechas de siembra fueron el 31 de octubre en 1990 y el 30 de octubre en 1991. Los fertilizantes fueron aplicados a la siembra, pero en el caso del nitrógeno se aplicó la mitad a la siembra y la otra mitad al primer aporque que se realizó a los 58 y 67 días después de la siembra en 1990-91 y 1991-92, respectivamente. Los fertilizantes comerciales utilizados en ambas campañas fueron la úrea $(46 \%$ de $\mathrm{N})$, el superfosfato triple $\left(46 \%\right.$ de $\left.\mathrm{P}_{2} \mathrm{O}_{5}\right)$ y el cloruro de potasio $\left(60 \%\right.$ de $\left.\mathrm{K}_{2} \mathrm{O}\right)$. El primer año se utilizó semilla certificada para ambas variedades y para el segundo año se utilizaron tubérculo-semillas provenientes del ensayo del primer año. 
Tabla 1. Tratamientos y niveles de fertilización evaluados en las campañas 1990-91 y 1991-92.

\begin{tabular}{cccc}
\hline Tratamientos & $\mathrm{N}-\mathrm{P}-\mathrm{K}(\mathrm{Kg} . / \mathrm{ha})$ & Tratamientos & $\mathrm{N}-\mathrm{P}-\mathrm{K}(\mathrm{Kg} . / \mathrm{ha})$ \\
\hline 1. & $0-0-0$ & 11. & $160-160-0$ \\
2. & $0-80-0$ & 12. & $160-240-0$ \\
3. & $0-160-0$ & 13. & $240-0-0$ \\
4. & $0-240-0$ & 14. & $240-80-0$ \\
5. & $80-0-0$ & 15. & $240-160-0$ \\
6. & $80-80-0$ & 16. & $240-240-0$ \\
7. & $80-160-0$ & 17. & $160-160-60$ \\
8. & $80-240-0$ & 18. & $160-160-120$ \\
9. & $160-0-0$ & 19. & $240-160-60$ \\
10. & $160-80-0$ & 20 & $240-160-120$ \\
\hline
\end{tabular}

Las variables agronómicas observadas durante el ciclo del cultivo fueron: porcentaje de emergencia, número de tallos principales por planta, porcentaje de cobertura del suelo por el follaje (CF) y el rendimiento, que incluyó el número y peso de los tubérculos separados por tamaño. Los tamaños considerados fueron: IV (<35 mm), III $(35-45 \mathrm{~mm})$, II $(45-55 \mathrm{~mm})$ y I (> 55 $\mathrm{mm}$ ). La cobertura del suelo por el follaje fue tomada cada 15 días en las mismas plantas marcadas en cada parcela mediante dos lecturas que incluyeron tres plantas. La evaluación se realizó mediante un marco de $0.9 \mathrm{~m} x$ $0.7 \mathrm{~m}$ dividido en 100 cuadros iguales. El bastidor se colocó encima de las plantas marcadas para observar y contar el número de cuadros llenados por más de $50 \%$ con follaje verde $(1,2,3)$. La Duración de la Cobertura del Suelo por el Follaje (DCF) se calculó sumando los promedios de porcentaje de suelo cubierto por el follaje verde (CF) de dos observaciones sucesivas y multiplicándola por el número de días entre dichas observaciones (7). Estas sumas obtenidas cada dos observaciones sucesivas, se sumaron para obtener una DCF total por tratamiento.

Las muestras de suelos fueron recolectadas en las parcelas de experimentación antes de la siembra para determinar el nivel de fertilidad inicial (Tabla 2). Después de la cosecha, se tomaron otras muestras de suelos en las parcelas de los tratamientos $1,4,6,7,11,13,15$, y 17 para analizar el nivel de fertilidad residual, en función a la variedad sembrada y los niveles de $\mathrm{N}-\mathrm{P}_{2} \mathrm{O}_{5}-\mathrm{K}_{2} \mathrm{O}$ aplicados. 
Tabla 2. Resultados del análisis químico y físico del suelo antes de la siembra para las campañas 1990-91 y 1991-92.

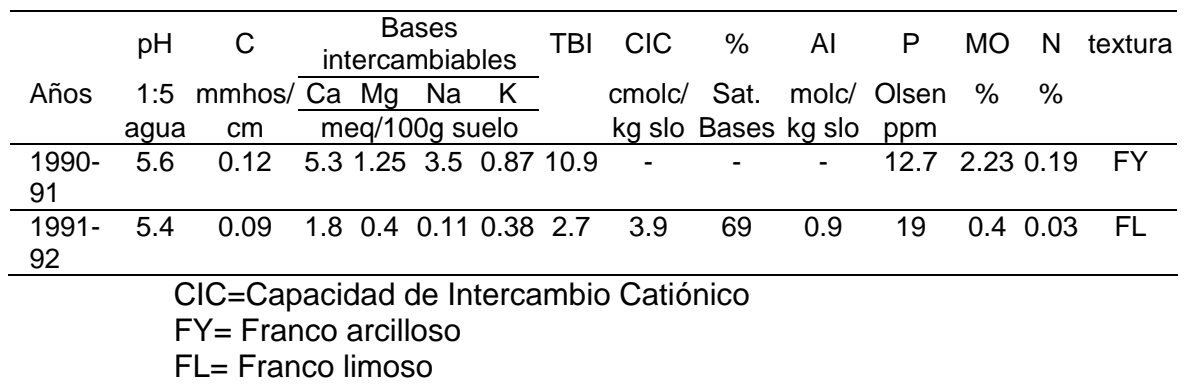

\section{Resultados y Discusión}

En forma general, se observó que la aplicación de nitrógeno en dosis superiores a $160 \mathrm{Kg} . / \mathrm{ha}$, especialmente cuando fue aplicado sin fósforo, retrasó la emergencia de las dos variedades que necesitaron tres semanas más para llegar a una emergencia de 90\% de plantas en 1991-92. Durante el segundo año, la sequía que se presentó a principios de la campaña retrasó más la emergencia de la variedad Rosita que la de Waych'a. Esta diferencia se notó en las primeras lecturas a los 30 días después de la siembra (DDS) con datos de emergencia con un promedio de $35 \%$ para Rosita y $52 \%$ para Waych'a. Esta diferencia desapareció con las lluvias de fines de diciembre (51 DDS).

El número de tallos por planta fue ligeramente superior para la variedad Rosita frente a la variedad Waych'a con efectos reducidos de los niveles de fertilización mineral (Tabla 3).

La respuesta de ambas variedades fue muy marcada a la aplicación de nitrógeno y fósforo pero la aplicación de potasio no tuvo un efecto significativo sobre el rendimiento en las dos campañas. En condiciones favorables de precipitaciones del año 1990-91, Rosita tuvo una mejor producción que Waych'a y su rendimiento siguió incrementando hasta niveles de $240 \mathrm{~kg}$./ha de nitrógeno y fósforo para alcanzar 45 t/ha (Figura 2a). Waych'a no alcanzó niveles de producción tan altos y su respuesta es mucho

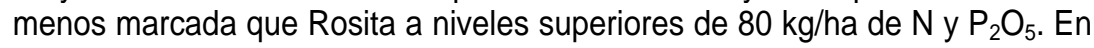
la segunda campaña, la curva de respuesta de rendimiento a niveles de nitrógeno llegó a su máximo para el nivel de 160 kg/ha para ambas variedades. 
La respuesta a fósforo fue notoria para el nivel $80 \mathrm{Kg} . / \mathrm{ha}$ con un leve incremento de rendimiento para los niveles superiores hasta $240 \mathrm{Kg}$./ha (Figura 3a). Cabe mencionar que el rendimiento de la variedad Rosita, en la campaña 1991-92, fue mucho más afectado por las condiciones de fertilidad más bajas de suelo y por la sequía que se presentó a principios de campaña; su producción más alta fue equivalente a 15 t/ha mientras la Waych'a llegaba a niveles de 35 t/ha. Además, considerando que la semilla utilizada el segundo año provenía del ensayo del año anterior, el bajo rendimiento de Rosita puede explicarse por una degeneración más rápida debida a nematodos y condiciones de almacenamiento.

Tabla 3. Efectos de los niveles de nitrógeno sobre el número de tallos por planta durante las dos campañas del ensayo.

\begin{tabular}{lllllllll}
\hline & \multicolumn{4}{c}{$\begin{array}{c}\text { Número de tallos por planta } \\
\text { Vampaña }\end{array}$} & \multicolumn{3}{c}{ Variedad Rosita } & \multicolumn{5}{c}{$\mathrm{N}(\mathrm{kg} / \mathrm{ha})$} \\
\cline { 2 - 9 } & \multicolumn{4}{c}{$\mathrm{N}(\mathrm{kg} / \mathrm{ha})$} & & \\
\hline \multirow{2}{*}{$1990-91$} & 2.01 & 1.9 & 1.95 & 1.90 & 1.61 & 1.74 & 1.93 & 1.82 \\
$1991-92$ & 2.39 & 2.61 & 2.51 & 2.50 & 2.12 & 2.23 & 2.22 & 2.3 \\
\hline
\end{tabular}

1990-91 DMS $0.05=0.25$ C.V.35.5\%

1991-92 DMS $0.05=0.25$ C.V. $26.8 \%$ 

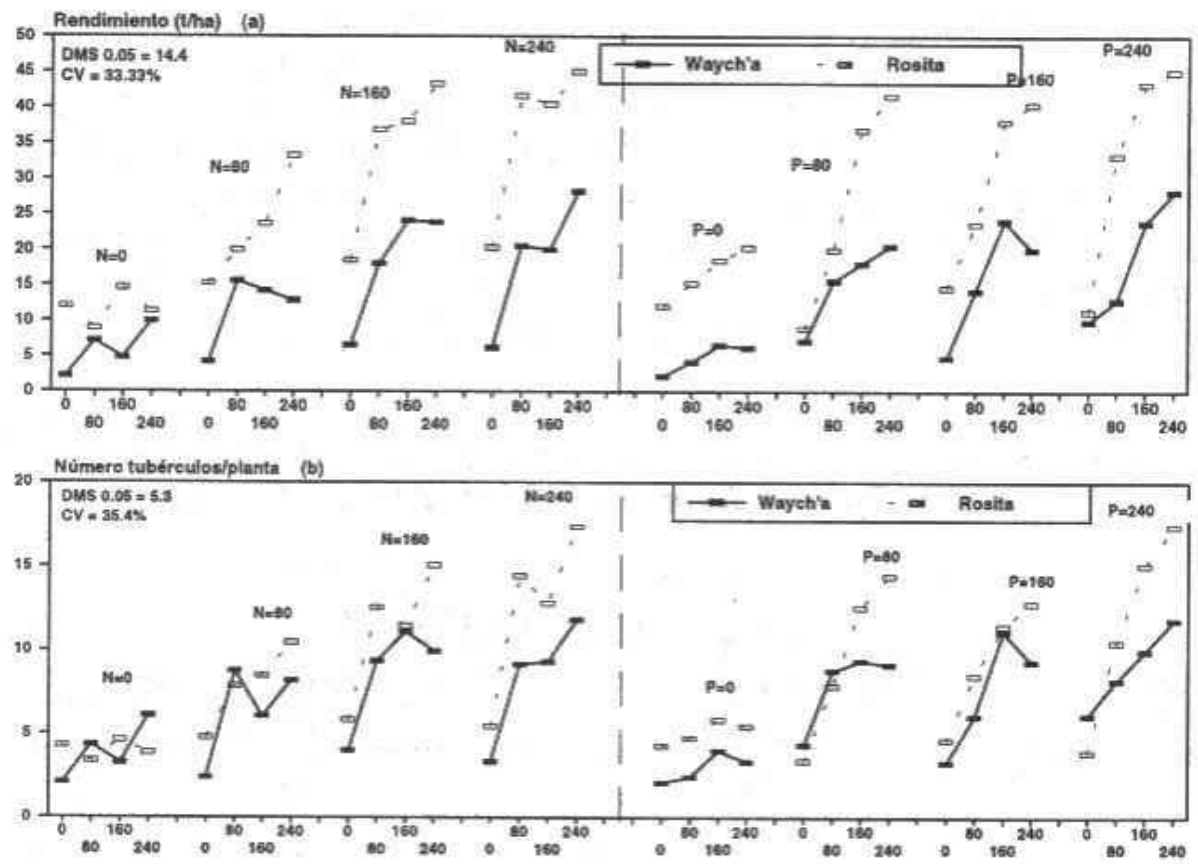

Figura 2. Efecto de la combinación de cuatro niveles de nitrógeno y de fósforo en el a) rendimiento y b) número de tubérculos por planta, de las variedades Waych'a y Rosita. Toralapa, Cochabamba, 1990-91.

El efecto de los niveles de nitrógeno y fósforo sobre el número de tubérculos por planta sigue la misma tendencia que los rendimientos, lo que indica que la respuesta de ambas variedades a los niveles de fertilización se debe más al incremento del número de tubérculos, que al incremento del tamaño de los tubérculos. Esto se nota especialmente en la campaña 1990-91, en la cual el peso promedio de ningún tamaño, fue afectado por los niveles de nitrógeno y fósforo. En la campaña 1991-92, la respuesta en rendimiento de las dos variedades a los niveles de fertilización se debió al incremento del número de tubérculos como al incremento del peso promedio sobre todo de los tamaños uno y dos. En el caso de Waych'a el número de tubérculos por planta fue más elevado en 1991-92 y está en relación con los rendimientos más altos observados durante esta campaña. 

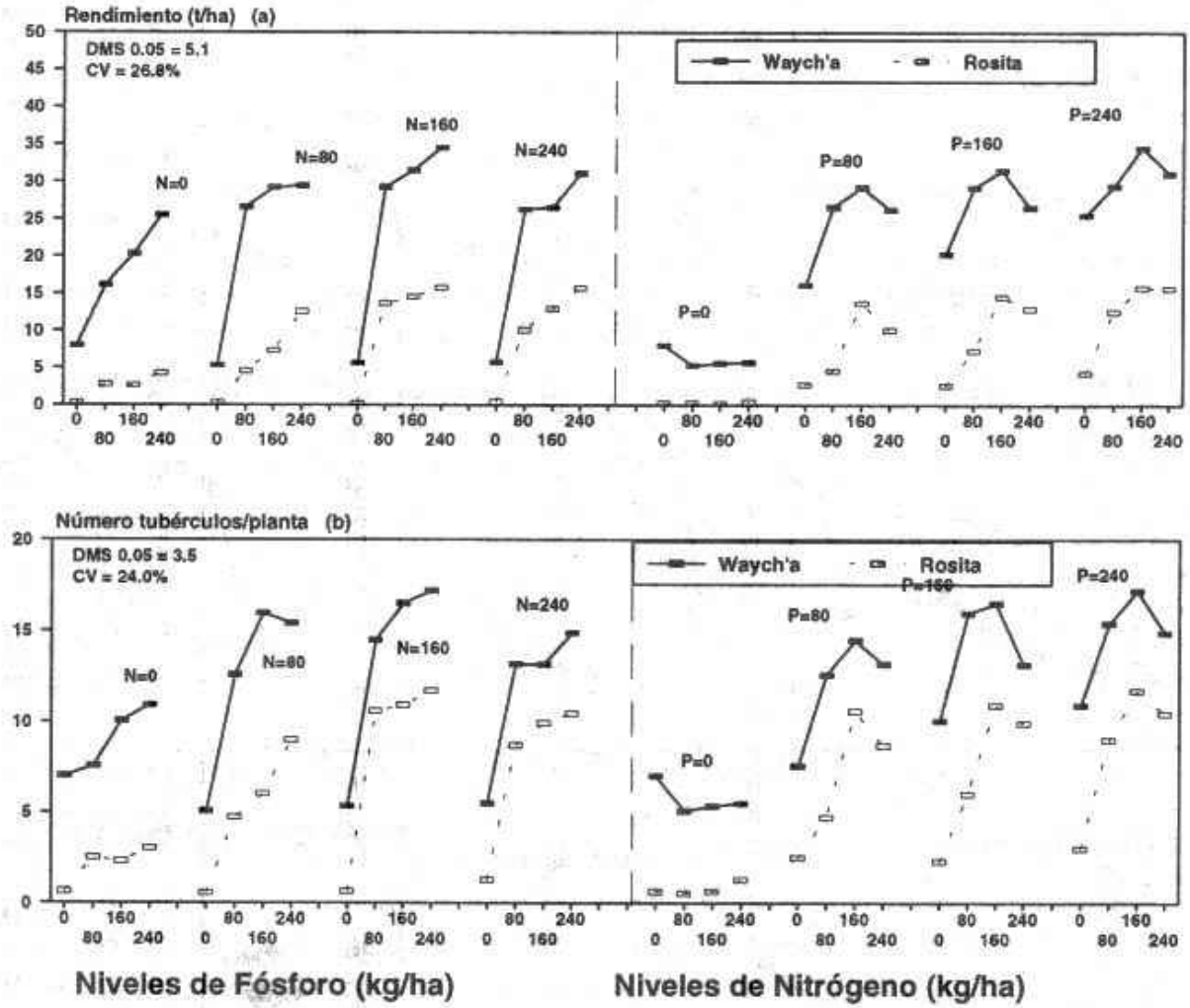

Figura 3. Efecto de la combinación de cuatro niveles de nitrógeno $y$ de fósforo en a) rendimiento y el b) número de tubérculos por planta, de las variedades Waych'a y Rosita. Toralapa, Cochabamba 1991-92. 

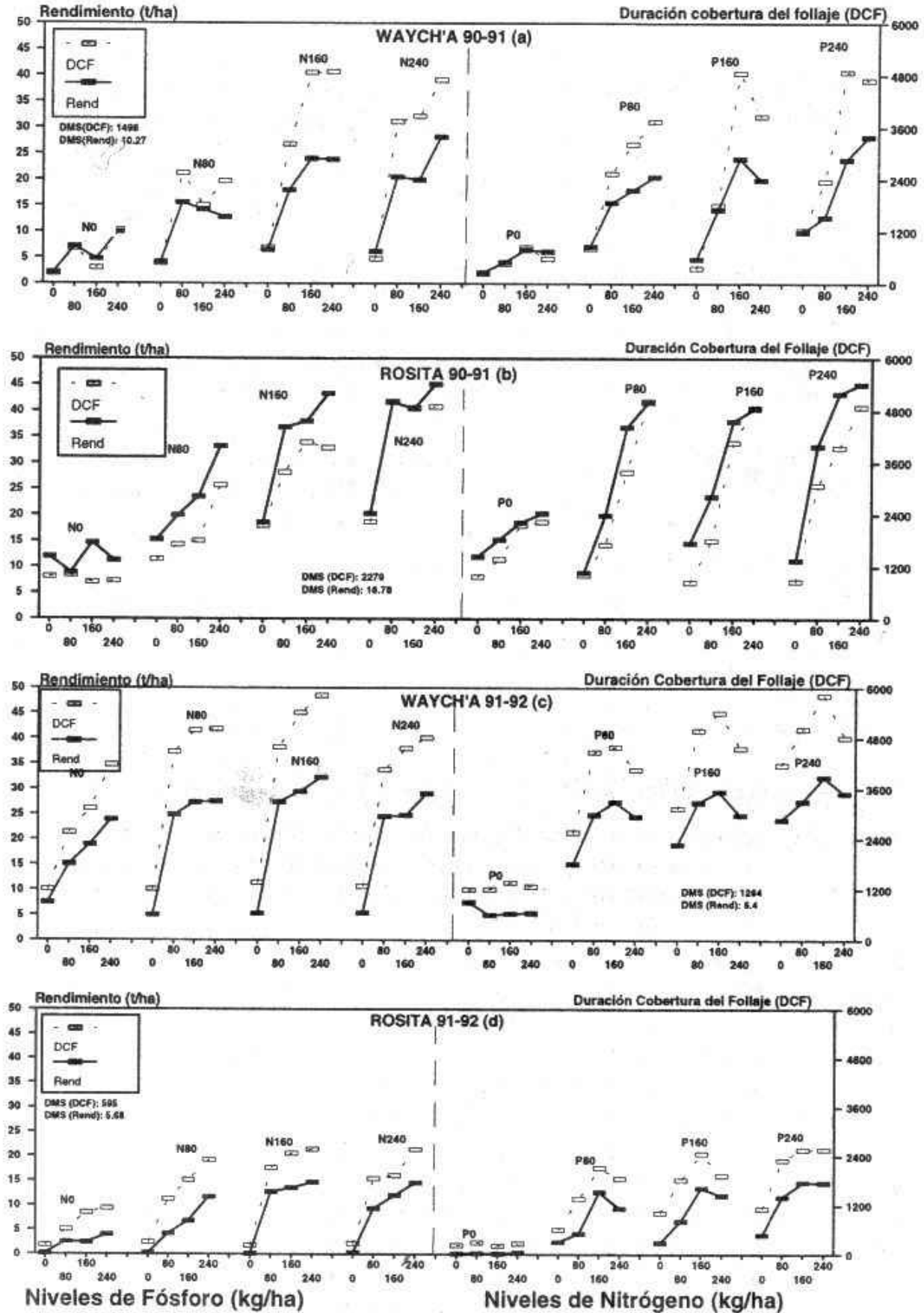

Figura 4. Efecto de la combinación de cuatro niveles de nitrógeno y de fósforo sobre la Duración de Cobertura del Suelo por el Follaje (DCF) y el Rendimiento (t/ha), de las variedades Waych'a y Rosita. Toralapa, Cochabamba, 1991-92. 
Durante la primera campaña ambas variedades tuvieron un ciclo vegetativo de 160 días mientras que en 1991-92 el ciclo fue afectado por heladas a partir de los 141 días después de la siembra. Los niveles de fertilización probados influyeron mucho en el desarrollo del follaje de las dos variedades. En las Figuras 4 a, b, c, y d comparamos la duración de cobertura de suelo por el follaje (DCF) con el rendimiento para analizar las respuestas de las dos variedades a los niveles de fertilización mineral. En la campaña 1990-91, las dos variedades tuvieron una DCF similar, pero Rosita tuvo un rendimiento más alto, lo que indica una mejor eficiencia de esta variedad para utilizar la radiación interceptada en la producción de tubérculos. La comparación entre las dos campañas muestra que para la variedad Waych'a, los valores de DCF y el rendimiento presentan la misma tendencia, mientras que para la variedad Rosita hay una diferencia marcada entre las dos campañas. Bajo condición de estrés, en este caso el estrés hídrico, de baja fertilidad del suelo y de baja calidad de semilla, la variedad Rosita fue más afectada en el desarrollo del follaje con una consecuencia directa en la producción de tubérculos.

Las relaciones entre la duración de cobertura del suelo por el follaje y el rendimiento en función a los niveles de N y P durante las dos campañas, confirman estas diferencias de comportamiento de las variedades (Figura 5). Pero se observa que las líneas de regresión son similares en ambas campañas. Esto indica que cada variedad mantiene una eficiencia similar del uso de la radiación para la producción de tubérculos en ambas campañas. La pendiente de la línea de regresión fue más elevada para Rosita que Waych'a, lo que indica una mayor eficiencia de radiación. La mejor respuesta de Rosita a niveles de $\mathrm{N}$ y $\mathrm{P}$ se debe a su mejor desarrollo foliar (Figura $4 \mathrm{~b}$ ) y a una mayor eficiencia del uso de la radiación para la producción de tubérculos. Como en la segunda campaña, el desarrollo foliar de Rosita fue afectado (Figura 4d) por diferentes tipos de estrés, su rendimiento bajó, a pesar de mantener una buena eficiencia del uso de radiación. Estos datos indican que el desarrollo de la planta está más afectado por las condiciones de cultivo, mientras que la eficiencia del uso de radiación es más estable. Waych'a presenta un desarrollo de planta menos viable que Rosita bajo condiciones agroclimáticas diferentes, lo que le confiere más estabilidad de rendimiento.

Los niveles de potasio combinados con nitrógeno y fósforo, no tuvieron efecto sobre el número de tubérculos ni el rendimiento durante la primera campaña. Durante la segunda campaña, la aplicación de potasio contribuyó a aumentar el número de tubérculos y el rendimiento de ambas variedades, pero la diferencia observada no fue significativa. 
El nivel más bajo de potasio en el suelo en 1991-92 puede explicar la diferencia de respuesta de las variedades entre las dos campañas.

La fertilidad del suelo en las parcelas de la campaña 1991-92 fue menor, pero $\mathrm{P}$ era más alto, en comparación a la fertilidad de los suelos de la campaña 1990-91 (Tabla 2). Esta diferencia de fertilidad por una parte y principalmente la sequía a principios de campaña, afectaron más a la variedad Rosita, de menor desarrollo radicular, que la variedad Waych'a.

Los valores de concentración de los elementos minerales en el suelo después de la cosecha (DC) en las dos variedades, no variaron respecto a los valores observados antes de la siembra excepto para el contenido de fósforo, que fue el único elemento cuya concentración varió en función de los tratamientos y las variedades estudiadas. El mayor residuo de fósforo en el suelo en el segundo año, con relación al primer año, se debe a la menor humedad del suelo, que no permitió una óptima asimilación de este elemento. De manera lógica, en las dos campañas se observó una mayor acumulación de fósforo, elemento menos móvil entre otros nutrientes (4), en los tratamientos con los niveles mayores de aplicación. Además se observó que Waych'a tiende a usar menos fósforo que Rosita (Figura 6). Esto se observa especialmente en la primera campaña donde Waych'a produjo menos que Rosita, lo que explica también el menor uso de fósforo. Este aspecto necesita más investigación.

\section{Conclusiones}

La comparación de las dos variedades durante las dos campañas mostró un comportamiento diferente según las condiciones de suelo y de precipitaciones. La variedad Waych'a (spp. andigena) presentó una mayor estabilidad de desarrollo del cultivo y de rendimiento frente a los factores abióticos que pueden afectar al cultivo, tales como la fertilidad del suelo, calidad de semilla y factores abióticos y bióticos. No se estudió específicamente el efecto de los nematodos en estos ensayos pero, por la presencia de $N$. aberrans y Globodera spp. en la Estación Experimental Toralapa, es también posible que estos parásitos hayan afectado más a la variedad Rosita que Waych'a, la cual ha demostrado una buena tolerancia a N. aberrans (9). 


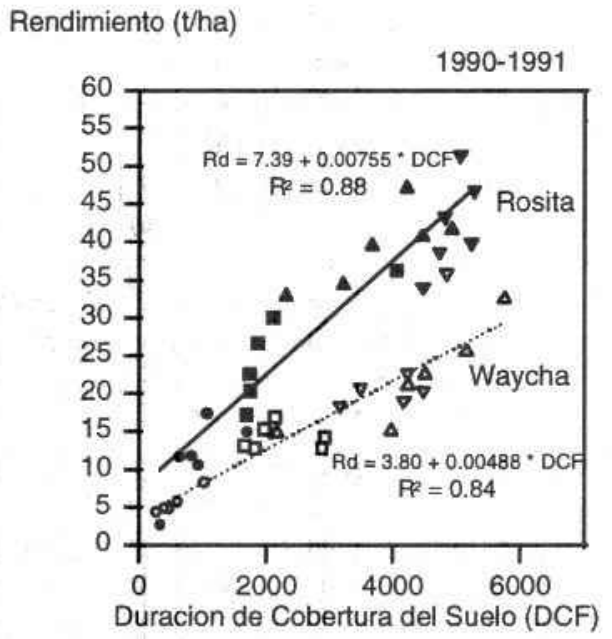

Rendimiento (t/ha)

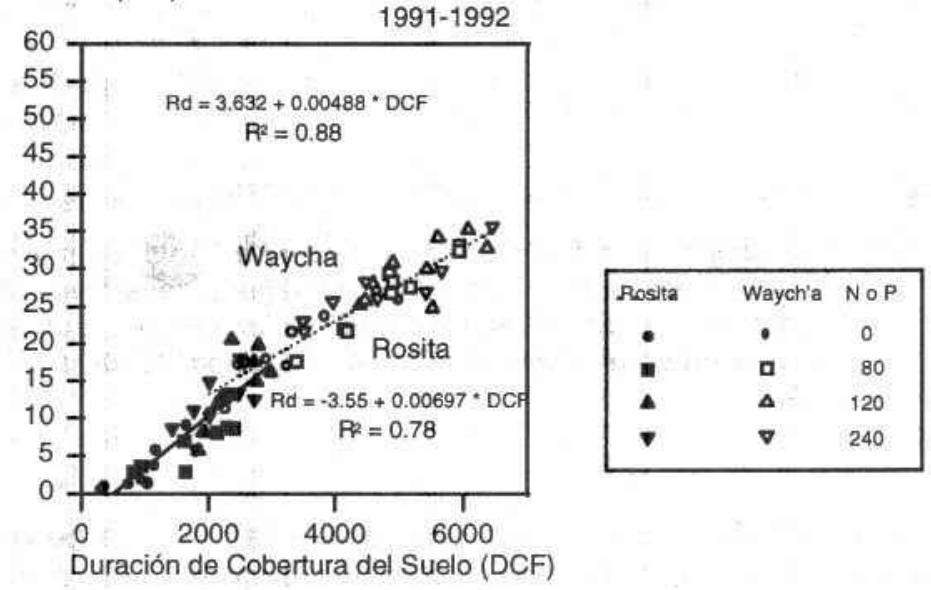

Figura 5. Relación entre la duración de cobertura del suelo por el follaje y el rendimiento en función de los niveles de nitrógeno (0 a $240 \mathrm{Kg} . / \mathrm{ha}$ ) en dos campañas (1990-91 y 1991-92) 

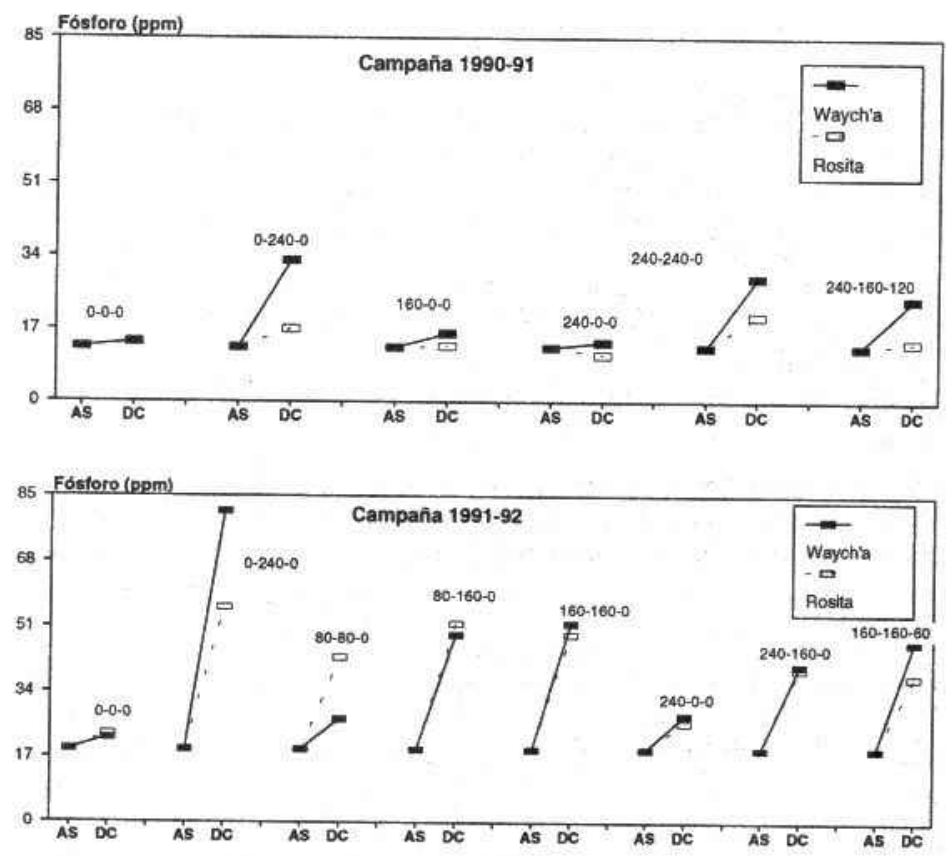

Figura 6. Variación del contenido de fósforo asimilable (ppm) en el suelo antes de la siembra (AS) y después de la cosecha (DC), en algunos niveles de fertilización mineral, aplicados a las variedades Waych'ay Rosita. Toralapa, 1990-91, 199192.

En ambas campañas se observó una respuesta marcada de las dos variedades a la aplicación de fósforo y nitrógeno. En cuanto al fósforo, hubo una respuesta positiva de ambas variedades hasta niveles de $240 \mathrm{Kg}$./ha pero el óptimo pareció situarse alrededor de $80 \mathrm{Kg}$./ha. El grado de respuesta a niveles más altos fue más reducido. El comportamiento de las dos variedades fue similar los dos años. En cuanto a la respuesta a niveles de nitrógeno, hubo una diferencia de comportamiento entre las variedades y las dos campañas. Para la variedad Waych'a el nivel óptimo estuvo alrededor de $80 \mathrm{Kg}$./ha pero varió entre campañas. En la primera campaña hubo una respuesta marcada hasta niveles de $160 \mathrm{Kg}$./ha cuando se incrementaron los niveles de fósforo hasta 160 y $240 \mathrm{Kg}$./ha. En la segunda campaña la respuesta óptima a la aplicación de nitrógeno se mantuvo para el nivel de 80 $\mathrm{Kg} . /$ ha. Para la variedad Rosita, hubo una respuesta marcada hasta niveles de nitrógeno de 160 kg./ha. en ambas campañas. 
La aplicación de niveles de 60 y $120 \mathrm{~kg} / \mathrm{ha}$ de potasio no tuvo un efecto significativo sobre el rendimiento, pero el segundo año, por el nivel más bajo de potasio en el suelo, se observó un efecto limitado en el número de tubérculos y el rendimiento de las dos variedades.

El efecto de los niveles de nitrógeno y fósforo sobre el número total de tubérculos siguió la misma tendencia que la respuesta observada para el rendimiento.

La relación entre la duración de cobertura del suelo por el follaje (DCF) y el rendimiento confirma la respuesta de la variedad Rosita a niveles más altos de fertilización, sobre todo de nitrógeno, frente a la variedad Waych'a (Figura 5). La pendiente más alta de la línea de regresión para Rosita indica una mayor eficiencia de esta variedad para utilizar la radiación interceptada en la producción de tubérculos. Faltan datos de materia seca de tubérculos para poder comparar la eficiencia real de producción de ambas variedades. Considerando que la eficiencia de uso de la radiación interceptada es relativamente estable para cada variedad, el rendimiento depende sobre todo del desarrollo del follaje de las plantas. El desarrollo de Waych'a fue más estable que el de Rosita frente a los factores adversos que se presentaron, sobre todo en la segunda campaña, lo que le confiere una mayor estabilidad de rendimiento que Rosita. Esta observación implica la importancia de concebir estrategias de fertilización, en función a las condiciones agroclimáticas y la clase de genotipo que se siembra. Los datos de análisis de suelo antes de la siembra y después de la cosecha parecen indicar que Waych'a extrae menos fósforo del suelo que Rosita. Se necesitaría realizar análisis de absorción de fósforo para confirmar esta observación.

Estas observaciones y la estabilidad de producción observada para la variedad Waych'a en ambas campañas demuestran el valor que tiene esta variedad para las condiciones agroclimáticas de producción diversas y variables que se observan en Solivia. Estas características, además de las calidades culinarias de esta variedad, explican la importancia que tiene para los agricultores en muchas zonas de producción de papa en Bolivia.

Los resultados de estos ensayos sirven de base para estudios de las estrategias de fertilidad de suelos en el contexto de los sistemas de producción de los agricultores, en combinación con enmiendas orgánicas y bajo diferentes condiciones agroclimáticas. 
Además de un conocimiento de los sistemas de producción de las diferentes zonas de producción de papa, hay una necesidad de contar con bases de datos de suelos y de clima para establecer estrategias de fertilidad de suelos adaptadas a las condiciones locales. Considerando la información ya disponible al respecto, hay que sistematizar esta información para poder utilizarla en forma eficiente. Esto se puede concretar mediante el uso de modelos de simulación que permitirán, una vez calibradas a las condiciones locales, extrapolar resultados y recomendaciones a las diversas zonas agroecológicas donde se cultiva la papa.

Este trabajo fue ejecutado dentro del marco del convenio del Programa de Investigación de la Papa, PROINPA, que fue establecido entre la Secretaría Nacional de Agricultura y Ganadería de Solivia, la Cooperación Suiza al Desarrollo (COSUDE) y el Centro Internacional de la Papa (CIP).

\section{Referencias Bibliográficas}

1. Bean, J.N. 1984. Light interception and leaf growth. Potato Research 27:313.

2. Burstall, L; Harris, P.M.. 1983. The estimation of percentage light interception from leaf area index and percentage ground cover in potatoes. Journal of Agriculture Science 100:241-244.

3. Centro Internacional de la Papa (CIP). s/f. Método sencillo para interpretar el crecimiento y rendimiento del cultivo de papa. Lima, Perú. p. 7-9.

4. Cooke, G. W. 1992. Fertilización para rendimientos máximos. Compañía Editorial Continental, S. A. de C. V. México D. F., México. 383 p.

5. Espinoza, G. 1990. Niveles de fertilización en el cultivo de papa en el Departamento de Cochabamba. En. Seminario Nacional sobre fertilidad de suelos y uso de fertilizantes en Bolivia (1., 1990, Santa Cruz, Bol.). IBTA-FAO-CIAT. Santa Cruz, Bolivia, p. 122-133.

6. Herbas, J. 1990. Evaluación agronómica de la aplicación de abonos orgánicos y fertilizantes químicos en ensayos a largo plazo en la Tamborada. En. Seminario Nacional sobre fertilidad de suelos y uso de fertilizantes en Bolivia. IBTA-FAO-CIAT. Santa Cruz, Bolivia, p. 354-365 
7. Levy, D.; Livesku, L; Van der Zaag, D.E. 1986. Double cropping of potatoes in a semiarid environment: The association of ground cover with tuber yield. Potato Research 29:437-449.

8. Monasterios, T. 1967. Respuesta de la papa a los fertilizantes en la Región Altiplánica de Bolivia. Estación Experimental Toralapa. Ministerio de Agricultura. Cochabamba, Bolivia. 30 p.

9. Ramos, J. 1994. Efecto de la humedad del suelo y la infección de Nacobbus aberrans en el comportamiento agrofisiológico de los cultivares Alpha (S. tuberosum) y Waych'a (S. andigena). Tesis Ingeniero Agrónomo. Universidad Mayor de San Simón, Cochabamba, Bolivia. 